\section{Re: Care of Patients Who Are Worried about Mercury Poisoning from Dental Fillings}

To the Editor: In the Research Letter by Vearrier and Greenberg, ${ }^{1}$ the authors elegantly describe a 37-yearold woman with clinically suspected overexposure to mercury from her mercury-containing dental amalgam fillings. She also had thyroid dysfunction. In this context, concerns regarding the risk of mercury amalgam were raised by her wellness doctor after the administration of the intravenous chelating agent dimercaptosuccinic acid, resulting in a marked increase of urinary excretion of mercury. ${ }^{1}$ Although the authors presented data indicating that the urinary mercury levels during re-evaluation were within the normal reference range $(<4 \mu \mathrm{g} / \mathrm{L}$; upper limit value, $<20 \mu \mathrm{g} / \mathrm{L}),{ }^{1}$ we are surprised that the authors did not include the results of mercury concentrations in whole blood that could have confirmed the absence of continuous overexposure to mercury from her dental amalgam fillings.

This important point is not well understood yet. Previous studies have demonstrated that there is a significant correlation between mercury-containing amalgam fillings and both plasma and blood concentrations of mercury in adults, and positive associations have been observed. ${ }^{2}$ Therefore, in addition to the urinary mercury levels as written by Vearrier and Greenberg, ${ }^{1}$ we suggest that blood and/or plasma level determinations also should be performed as a screen for patients with a suspected adverse event caused by mercury-containing dental amalgam fillings. Second, the findings of a preliminary epidemiologic and allergologic study of adverse reactions to mercury amalgam provides evidence that the $30.89 \%$ of 259 patients had an allergic reaction to mercury compounds, in aggregate. $^{3}$ In our experience ${ }^{4,5}$ and that of others, ${ }^{6}$ the thyroid would seem to be a primary target organ of mercury released from mercury amalgams. Because of these reference data, ${ }^{4-6}$ the wellness physician may have supposed a link between his patient's thyroid disorder and her dental amalgam fillings. Reassuring our patients that they are not being poisoned by mercury from their amalgam can be very helpful because it is a very rare event.

However, we should provide patients and physicians with correct information about the potential of toxicology tests, including the patch test, for adverse events potentially related to mercury-containing den- tal amalgams. ${ }^{3}$ The case study of Vearrier and Greenberg $^{1}$ reminds us that chelation therapy is not needed when the toxicological tests results of a patient with suspected overexposure to mercury amalgam are below the reference range.

Gianpaolo Guzzi

Italian Association for Metals and Biocompatibility Research, Milan, Italy gianpaolo_guzzi@fastwebnet.it

Lucia Brambilla

Operative Unit of Dermatology, Fondazione IRCCS Ca, Ospedale Maggiore Policlinico

Paolo D. Pigatto

Department of Technology for Health, Dermatological Clinic, IRCCS Galeazzi Hospital, University of Milan, Italy

\section{References}

1. Vearrier D, Greenberg MI. Care of patients who are worried about mercury poisoning from dental fillings. J Am Board Fam Med 2010;23:797-8.

2. Lorscheider FL, Vimy MJ, Summers AO. Mercury exposure from "silver" tooth fillings: emerging evidence questions a traditional dental paradigm. FASEB J 1995;9: 504-8.

3. Pigatto PD, Brambilla L, Ferrucci SM, Minoia C, Ronchi A, Guzzi G. Allergy and adverse reactions to dental amalgam: an epidemiological assessment 10th Congress of the European Society of Contact Dermatitis Sept 2010 ESCD-GERDA Strasbourg. Contact Dermatitis 2010; 63(Suppl 1):95-105.

4. Guzzi G, Grandi M, Cattaneo C, et al. Dental amalgam and mercury levels in autopsy tissues: food for thought. Am J Forensic Med Pathol 2006;27:42-5.

5. Pigatto PD, Marsili C, Brambilla L, Guzzi G. Cutaneous manifestations, thyroid disease, and dental amalgam. Presented at the 18th Congress of the European Academy of Dermatology and Venereology, Berlin, Germany, 7-11 October 2009; p. 1388.

6. Sterzl I, Prochazkova J, Hrda P, Matucha P, Bartova J, Stejskal V. Removal of dental amalgam decreases anti-TPO and anti- $\mathrm{Tg}$ autoantibodies in patients with autoimmune thyroiditis. Neuro Endocrinol Lett 2006;27:25-30.

7. Langworth S, Strömberg R. A case of high mercury exposure from dental amalgam. Eur J Oral Sci 1996;104:320-1.

doi: $10.3122 /$ jabfm.2011.04.110023

The above letter was referred to the author of the article in question, who offers the following reply. 